\title{
Charge Transfer at Large Scattering Angles in Asymmetric Ion-Atom Collisions
}

\author{
P. A. Amundsen \\ NORDITA, Copenhagen, Denmark ${ }^{*}$ \\ D. H. Jakubaßa-Amundsen \\ Physik-Department, Technische Universität München, Garching and GSI Darmstadt, Germany
}

Received December 28, 1981; accepted March 12, 1982

A general formula for the dependence of the electron capture probability on scattering angle in fast collisions is derived using the semiclassical zero impact parameter approximation. We apply the impulse approximation (SCIA) to perform numerical calculations of the capture probability from the $K$ and $L$ shell of $\mathrm{Ne}$ and $\mathrm{Ar}$ targets by protons and give a comparison with recent experiments.

\section{Introduction}

The measurement of cross-sections differential in the projectile scattering angle has contributed much to our understanding of excitation and ionisation mechanisms in ion-atom collisions. Also for charge transfer in fast and asymmetric collisions, experiments have been performed which provided some insight in the dependence of the capture probability on impact par. amter and collision energy [1-3]. These measurements have mostly been concerned with very small projectile scattering angles, and could well be explained within the semiclassical impulse approximation $[4,5]$. Thereby the internuclear motion was approximated by a classical, straight-line trajectory.

Recently, however, Horsdal Pedersen and Loftager [6] have measured electron capture by protons at large scattering angles, and they found a strong angular dependence of the capture probability in the case of $\mathrm{Ne}$ and $\mathrm{Ar}$ targets, while for a $\mathrm{He}$ target the capture probability turned out to be roughly constant. These experiments can no longer be described with a theory that neglects projectile deflection by using a straight-line path. We present in this paper a semi-classical higher-order theory for charge transfer which allows for a curved nuclear trajectory (Section 2). In order to extract the dependence of the capture probability on the scattering angle we make in Section 3 a partial wave analysis of the transition amplitude. Then we introduce the zero impact parameter semi-classical approximation $[7,8]$ where the classical nuclear trajectory is replaced by a "broken line". This means that the colliding nuclei are assumed to approach each other head-on and scatter through the given scattering angle at time $t=0$ (Section 4). This approximation is necessary to make numerical calculations feasible. In Section 5 the theory is specialized to the capture from the $K$ and $L$ shell into the projectile $1 s$ state, and the semi-classical broken-line impulse approximation is used to calculate the transition probabilities. Numerical results are given in Section 6 and compared with experimental data for collisions of protons

* Present address: Physik-Department T30, Technische Universität München, Garching, W. Germany. with $\mathrm{He}, \mathrm{Ne}$ and Ar targets. Concluding remarks follow (Section 7).

Atomic units ( $e=h=m=1$ ) are used throughout the paper, and the conventions concerning the angular momentum algebra follow Edmonds [9]. We also introduce the abbreviations $a=|\mathbf{a}|$ and $\hat{\mathbf{a}}=\mathbf{a} / a$ for the length and direction of the vector $\mathbf{a}$.

\section{General theory}

For definiteness we shall consider the transfer from a heavy target atom with an effective one-electron potential $V_{2}$ to a light projectile with a potential $V_{1}$. The formalism developed in this section is a generalisation of the straight-line theory as described by Jakubaßa-Amundsen and Amundsen [5] to an arbitrary nuclear trajectory. In a coordinate system centered at the target nucleus the Hamiltonian of the active electron is given by

$H=T+V_{2}(\mathbf{r})+V_{1}(\mathbf{r}-\mathbf{R})+V_{\mathbf{R}}$

where $T$ is the kinetic energy and

$V_{\mathrm{R}}=-\frac{M_{1}}{M_{1}+M_{2}} \mathbf{r} \cdot \ddot{\mathbf{R}}=\frac{1}{M_{2}} \frac{\partial V_{\mathrm{N}}(R)}{\partial R} \mathbf{r} \cdot \hat{\mathbf{R}}$

is the recoil potential which is present if the classical projectile path $\mathbf{R}(t)$ is not a constant-velocity straight-line path [10], $M_{1}$ and $M_{2}$ are the mass of the projectile and target, respectively, and $V_{\mathrm{N}}$ is the (spherical) internuclear potential.

The semi-classical transition amplitude in the prior form is then given by [11]

$a_{\mathrm{fi}}=-\mathrm{i} \int_{-\infty}^{\infty} \mathrm{d} t\left\langle\Phi_{\mathrm{f}}^{(-)}(t)\left|V_{1}+V_{\mathrm{R}}\right| \psi_{\mathrm{i}}^{\mathrm{T}}(t)\right\rangle$

where $\left|\psi_{\mathrm{i}}^{\mathrm{T}}(t)\right\rangle=\left|\psi_{\mathrm{i}}^{\mathrm{T}}\right\rangle \exp \left(-\mathrm{i} E_{\mathrm{i}}^{\mathbf{T}} t\right)$ is an eigenstate with energy $E_{\mathrm{i}}^{\mathrm{T}}$ of $H_{2}=T+V_{2}$ while $\left|\Phi_{\mathrm{f}}^{(-)}(t)\right\rangle$ is that solution of the full scattering problem which asympotically develops into an eigenstate of $H_{1}(t)=T+V_{1}(t)$, i.e., into a Galilei-transformed projectile eigenstate.

For an arbitrary time the asymptotic projectile state can be written in the following way

$$
\begin{aligned}
\left|\psi_{\mathrm{f}}^{\mathrm{P}}(t)\right\rangle & =\left|\mathrm{e}^{\mathrm{i} \dot{\mathbf{R}} \cdot \mathbf{r}} \psi_{\mathrm{f}}^{\mathrm{P}}(\mathbf{r}-\mathbf{R})\right\rangle \exp \left(-\mathrm{i} / 2 \int_{0}^{t} \dot{R}^{2} \mathrm{~d} t^{\prime}-\mathrm{i} E_{\mathrm{f}}^{\mathrm{P}} t\right) \\
& =\int \mathrm{d} \mathbf{k}|\mathbf{k}\rangle \mathrm{e}^{-\mathrm{i} \epsilon_{\mathrm{f}}(t)} \varphi_{\mathrm{f}}^{\mathrm{P}}(\mathbf{k}-\dot{\mathbf{R}}(t))
\end{aligned}
$$

where $\varphi_{\mathrm{f}}^{\mathrm{P}}$ is a momentum space projectile eigenstate of energy 
$E_{\mathfrak{f}}^{\mathrm{P}}$ (as seen in the projectile frame) and the phase is

$\epsilon_{\mathrm{f}}(t)=E_{\mathrm{f}}^{\mathrm{P}} t+\mathrm{k} \cdot \mathbf{R}(t)+\frac{1}{2} \int_{0}^{t} \dot{R}^{2} \mathrm{~d} t^{\prime}-\mathbf{R} \cdot \dot{\mathbf{R}}$

Since we are interested in asymmetric collisions we use a formulation that allows a systematic expansion of $\left|\Phi_{f}^{(-)}(t)\right\rangle$ in terms of the weaker of the two atomic potentials, $V_{1}$, while retaining the asymptotic behaviour of the wavefunction. Generalising from the straight-line case we find that $\left|\Phi_{f}^{(-)}(t)\right\rangle$ is a solution of

$$
\begin{aligned}
& \left|\Phi_{\mathfrak{f}}^{(-)}(t)\right\rangle=|\psi(t)\rangle+\int_{-\infty}^{\infty} \mathrm{d} t^{\prime} G_{2}^{(-)}\left(t, t^{\prime}\right) \\
& \quad\left[\left(V_{1}\left(t^{\prime}\right)+V_{\mathrm{R}}\left(t^{\prime}\right)\right)\left|\Phi_{\mathrm{f}}^{(-)}\left(t^{\prime}\right)\right\rangle-V_{1}\left(t^{\prime}\right)\left|\psi_{\mathrm{f}}^{\mathrm{P}}\left(t^{\prime}\right)\right\rangle\right]
\end{aligned}
$$

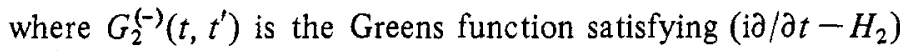
$G_{2}^{(-)}\left(t, t^{\prime}\right)=\delta\left(t-t^{\prime}\right)$. The potentials $V_{1}$ and $V_{\mathrm{R}}$ do not enter symmetrically into $(2.6)$ because $V_{\mathbf{R}}$ vanishes identically for $t \rightarrow \pm \infty$ and thus is not contained in the definition of the asymptotic state. Following Jakubaßa-Amundsen and Amundsen [5] the lowest-order solution $|\psi(t)\rangle$ is given by

$$
\begin{aligned}
|\psi(t)\rangle= & \int \mathrm{d} \mathbf{k} \frac{1}{2 \pi} \int_{-\infty}^{\infty} \mathrm{d} t^{\prime} \int_{-\infty}^{\infty} \mathrm{d} \omega \mathrm{e}^{-\mathrm{i} \omega t} \exp \left(-\mathrm{i}\left(\epsilon_{\mathrm{f}}\left(t^{\prime}\right)-\omega t^{\prime}\right)\right) \\
& \varphi_{\mathrm{f}}^{\mathrm{P}}\left(\mathbf{k}-\dot{\mathbf{R}}\left(t^{\prime}\right)\right)|\mathbf{k}, \omega\rangle^{\mathrm{T}}
\end{aligned}
$$

where $|\mathbf{k}, \omega\rangle^{T}$ is an off shell target continuum state of energy $\omega$.

While for a straight-line path, $R=(b, 0, v t)$, where $b$ is the impact parameter and $v$ the collision velocity, the $t^{\prime}$ and $\omega$ integrals can be carried out immediately, a similar reduction is not possible for a general projectile path, like a hyperbolic Kepler orbit. Thus the Coulomb deflection problem turns out to be much more complicated in the present case than for firstorder theories for excitation and ionisation, where one only has to change the projectile path dependence of $V_{1}(\mathbf{r}-\mathbf{R})$ in the transition matrix element [12].

Equation (2.7) can be approximated by making use of the fact that the main contribution to the $\omega$ integral comes from values of $\omega$ around the point of stationary phase:

$\omega_{0}\left(t^{\prime}\right)=\frac{\partial \epsilon_{\mathrm{f}}\left(t^{\prime}\right)}{\partial t^{\prime}}=E_{\mathrm{f}}^{\mathrm{P}}+\mathbf{k} \cdot \dot{\mathbf{R}}\left(t^{\prime}\right)-\frac{1}{2} \dot{R}^{2}\left(t^{\prime}\right)-\mathbf{R}\left(t^{\prime}\right) \cdot \ddot{\mathbf{R}}\left(t^{\prime}\right)$

When $|\mathbf{k}, \omega\rangle^{\mathbf{T}}$ is taken outside the $\omega$ integral at $\omega=\omega_{0}$, the $\omega$ and $t^{\prime}$ integrals in eq. (2.9) become trivial, yielding

$|\psi(t)\rangle=\int \mathrm{d} \mathbf{k} \mathrm{e}^{-\mathrm{i} \epsilon_{\mathrm{f}}(t)} \varphi_{\mathrm{f}}^{\mathrm{P}}(\mathbf{k}-\dot{\mathbf{R}}(t))\left|\mathbf{k}, \omega_{0}\right\rangle^{\mathrm{T}}$

which is a direct generalisation of the straight-line result [5]

If $\left|\Phi_{f}^{(-)}(t)\right\rangle$ can be approximated by the first-order expansion term in $V_{1},|\psi(t)\rangle$ as given by eq. (2.9), the transition amplitude finally becomes

$$
\begin{aligned}
a_{\mathrm{fi}}= & -\mathrm{i} \int_{-\infty}^{\infty} \mathrm{d} t \int \mathrm{d} \mathbf{k} \varphi_{\mathrm{f}}^{\mathrm{P} *}(\mathbf{k}-\dot{\mathrm{R}}(t)) \exp \left(\mathrm{i}\left(\epsilon_{\mathrm{f}}(t)-E_{\mathrm{i}}^{\mathrm{T}} t\right)\right) \\
& { }^{\mathrm{T}}\left\langle\mathbf{k}, \omega_{0}\left|V_{1}(t)+V_{\mathbf{R}}(t)\right| \psi_{\mathrm{i}}^{\mathrm{T}}\right\rangle
\end{aligned}
$$

Here we have used the fact that the recoil-contribution is small and can be treated as a perturbation.

The significance of the difference between eqs. (2.7) and (2.9) may loosely be interpreted as follows: The electron, being ejected into the target state $|\mathbf{k}, \omega\rangle^{\mathrm{T}}$ at time $t$ finds itself in a (travelling) projectile state at a different time $t^{\prime}$. The approximate result, eq. (2.9), neglects this time-delay. We have not been able to evaluate the transfer amplitude based on eq. (2.7) directly, so in the following sections we shall take eq. (2.10) as our starting point. It should be noted, however, that although we expect the stationary phase argument leading to eq. (2.9) to be valid in most situations, there may exist particular kinematical situations, where the off-shell wavefunction $|k, \omega\rangle^{T}$ varies too rapidly with $\omega$ for this to be a good approximation.

\section{Partial wave decomposition}

In the following we shall assume that the capture takes place from a state of angular momentum quantum numbers $L_{\mathrm{i}}, M_{\mathrm{i}}$ and with radial wavefunction $R_{\mathbf{i}}$ to a projectile state of angular momentum $L_{\mathrm{f}}, M_{\mathrm{f}}$ and momentum space radial wavefunction $P_{\mathrm{f}}$. A partial wave expansion of the ionisation matrix element is straightforward, and yields

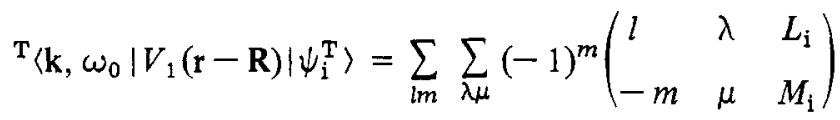

$$
\begin{aligned}
& \times\left\langle l\left\|Y_{\lambda}\right\| L_{\mathbf{i}}\right\rangle C_{l}(k) G_{\lambda, l}^{\mathbf{i}}\left(k, \omega_{0}, R\right) Y_{l}^{m}(\hat{\mathbf{k}}) Y_{\lambda}^{\mu^{*}}(\hat{\mathbf{R}})
\end{aligned}
$$

Here $\left\langle l\left\|Y_{\lambda}\right\| L_{i}\right\rangle$ is a reduced matrix element, while

$C_{l}(k)=i^{l} \frac{4 \pi}{k} \mathrm{e}^{\mathrm{i} \sigma_{l}(k)}$

$G_{\lambda, l}^{\mathbf{i}}\left(k, \omega_{0}, R\right)=\int_{0}^{\infty} r^{2} \mathrm{~d} r R_{l}^{*}\left(k, \omega_{0}, r\right) v_{\lambda}(r, R) R_{\mathrm{i}}(r)$

with $R_{l}\left(k, \omega_{0}, r\right)$ as the radial part of the target off-shell wavefunction, with a corresponding phase shift $\sigma_{l}(k)$. Furthermore, $v_{\lambda}(r, R)$ is the $2^{\lambda}$-pole component of $V_{1}$,

$V_{1}(\mathbf{r}-\mathbf{R})=\sum_{\lambda \mu} v_{\lambda}(r, R) Y_{\lambda}^{\mu^{*}}(\hat{\mathbf{R}}) Y_{\lambda}^{\mu}(\hat{\mathbf{r}})$

The recoil term can readily be incorporated in these expressions by adding a term to $v_{\lambda}$ for $\lambda=1$ from eq. (2.2).

For convenience we shall assume that the internuclear motion takes place in the $x-z$ plane. The classical path can then generally be written

$\mathbf{R}(t)=R(t)(\sin \alpha(t), 0, \cos \alpha(t))$

$\dot{\mathbf{R}}(t)=v(t)(\sin \beta(t), 0, \cos \beta(t))$

Since $v(t)$ in most cases will be a constant along the path, one can obtain a separation of the velocity-dependence of $\varphi_{\mathrm{f}}^{\mathrm{P}}(\mathbf{k}-\dot{\mathbf{R}})$ from the $k$-depencence by performing a time dependent rotation, so that the velocity becomes parallel to the $z$-axis. If the corresponding rotation operator is denoted by $D_{y}(\beta)$, we have, with $\mathrm{p}=\mathrm{k}-\dot{\mathbf{R}}(t)$

$$
\begin{aligned}
\varphi_{\mathrm{f}}^{\mathrm{P}}(\mathrm{p}) & =D_{y}(-\beta) P_{\mathrm{f}}\left(p^{\prime}\right) Y_{L_{\mathrm{f}}}^{M_{\mathrm{f}}}\left(\hat{\mathbf{p}}^{\prime}\right) \\
& =P_{\mathrm{f}}\left(p^{\prime}\right) \sum_{m^{\prime}} d_{m_{\mathrm{f}} M_{\mathrm{f}}}^{\left(L_{\mathrm{f}}\right)}(-\beta) Y_{L_{f}}^{m^{\prime}}\left(\Theta^{\prime}, \varphi_{k}^{\prime}\right)
\end{aligned}
$$

Here $\mathbf{p}^{\prime}=D_{y}(\beta) \mathbf{p}$ - and similarly for other vectors - so that $p^{\prime}=\left|\mathbf{k}^{\prime}-v \mathrm{e}_{\boldsymbol{z}}\right|$. Furthermore, $\left(\Theta_{k}^{\prime}, \varphi_{k}^{\prime}\right)$ are the polar angles of $\hat{\mathbf{k}}^{\prime}, d_{m M_{\mathbf{f}}}^{\left(L_{\mathrm{f}}\right)}$ is a rotation matrix element and $\sin \Theta^{\prime}=k^{\prime} \sin \Theta_{k}^{\prime} / p$. 
Making a similar transformation in eq. (3.1) and carrying out the integration over $\varphi_{k}^{\prime}$, we find after some manipulations that eq. (2.10) can be written

$$
\begin{aligned}
& a_{\mathrm{fi}}=-2 \pi \mathrm{i}(-1)^{M_{\mathrm{i}}} \sum_{l \lambda}\left\langle l\left\|Y_{\lambda}\right\| L_{\mathrm{i}}\right\rangle \int_{0}^{\infty} k^{2} \mathrm{~d} k C_{l}(k) \\
& \times \sum_{m_{1}, m, \mu}(-1)^{\mu}\left(\begin{array}{lll}
l & \lambda & L_{\mathrm{i}} \\
-m_{1} & \mu & m_{1}-\mu
\end{array}\right) d_{M_{\mathrm{i}}, m_{1}-\mu}^{\left(\mathrm{L}_{\mathrm{i}}\right)}(-\beta) \\
& \times d_{m M_{\mathrm{f}}}^{\left(L_{\mathrm{f}}\right)}(-\beta) \mathrm{i}^{\left|m_{1}-m\right|} Y_{\lambda}^{\mu^{*}}\left(\hat{\mathrm{k}}^{\prime}\right) \int_{-\infty}^{\infty} \mathrm{d} t \mathrm{e}^{\mathrm{i} \Omega_{0} t} \\
& \times \quad \int_{-1}^{1} \mathrm{~d}\left(\cos \Theta_{k}^{\prime}\right) Y_{L_{\mathrm{f}}}^{m^{*}}\left(\Theta^{\prime}, 0\right) Y_{l}^{m_{1}}\left(\Theta_{k}^{\prime}, 0\right) \\
& \times J_{\left|m_{1}-m\right|}\left(k R \sin \Theta_{k}^{\prime} \sin (\alpha-\beta)\right) G_{\lambda, l}^{\mathrm{i}}\left(k, \omega_{0}, R\right) \\
& \times \exp \left[i k R \cos \Theta_{k}^{\prime} \cos (\alpha-\beta)\right] \\
& \times P_{\mathrm{f}}^{*}\left(\sqrt{\left.k^{2}+v^{2}(t)-\cdots 2 k v(t) \cos \Theta_{k}^{\prime}\right)}\right.
\end{aligned}
$$

where

$\Omega_{0} t=\left(E_{\mathrm{f}}^{\mathrm{P}}-E_{\mathrm{i}}^{\mathrm{T}}\right) t+\frac{1}{2} \int_{0}^{t} v^{2}(t) \mathrm{d} t-v(t) \mathbf{R}^{\prime} \mathbf{e}_{\mathrm{z}}$

and $J_{m}$ is a Bessel function. In this expression the dependence on the scattering angle is still contained in a nontrivial way, being determined by the directions of $\mathbf{R}$ and $\dot{\mathbf{R}}$, i.e., $\alpha$ and $\beta$. We also see that much of the complications can be traced back to the expression (3.6), i.e., to the fact that the travelling projectile state has a complicated angular momentum structure.

\section{Zero impact parameter approximation}

The transfer amplitude, eq. (3.8), simplifies considerably when the internuclear vector is (anti-) parallel to the velocity vector. This is the case for the zero impact parameter ("broken line") approximation of Ciocchetti and Molinari [7]. As illustrated in Fig. 1, this approximation is obtained from eq. (3.5) by choosing

$\alpha(t)=\left\{\begin{array}{ll}\pi-\vartheta / 2, & t<0 \\ \vartheta / 2, & t>0\end{array} \quad \beta(t)= \begin{cases}-\vartheta / 2, & t<0 \\ \vartheta / 2, & t>0\end{cases}\right.$

where $\vartheta$ is the scattering angle, and the radial velocity is set equal to its asymptotic value, $v(t)=v$. Then the energy $\Omega_{0}=$ $E_{\mathrm{f}}^{\mathrm{P}}-E_{\mathrm{i}}^{\mathrm{T}}-v^{2} / 2$ becomes time-independent. This approximation is reasonable if the classical time delay from the slowing down and scattering of the nuclei is short compared to typical orbiting times of the active electron, and if the distance of closest

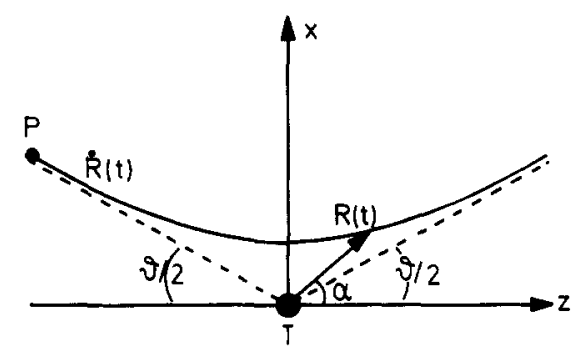

Fig. 1. Illustration of the approximation of the classical nuclear trajectory by a zero impact parameter broken-line path. approach between the two nuclei is much smaller than the electronic shell radius. These conditions are well satisfied in the case of high-energy charge transfer, and also the choice of $b=0$ is a grood approximation, as the large scattering angles correspond to very small impact parameters.

Quantum mechanically, the applicability of the brokenline approximation implies that the main contribution to the total transfer matrix element comes from regions of space where the nuclear wavefunctions are close to their asymptotic values. Then it can be shown that the zero impact parameter SCA and the quantal first-order Born approximation are equivalent, where the argumentation follows closely the theory for nuclear time-delay effects and their influence on the ionisation probability in asymmetric collisions [13].

With this approximation, eq. (3.8) simplifies considerably. Only terms with $\mu=0$ and $m_{1}=m$ survive, and using the product formula for rotation matrix elements, one arrives at:

$$
\begin{aligned}
& a_{\mathrm{fi}}=-\mathrm{i} \sqrt{\pi} \sum_{l \lambda}(-1)^{M_{\mathrm{i}}} \sqrt{2 \lambda+1}\left\langle l\left\|Y_{\lambda}\right\| L_{\mathrm{i}}\right\rangle \\
& \times \sum_{\Lambda M m}(2 \Lambda+1) d_{M 0}^{(\Lambda)}(\vartheta / 2)\left(\begin{array}{lll}
l & \lambda & L_{\mathrm{i}} \\
-m & 0 & m
\end{array}\right) \\
& \times\left(\begin{array}{ccc}
L_{\mathrm{f}} & L_{\mathrm{i}} & \Lambda \\
m & -m & 0
\end{array}\right)\left(\begin{array}{lll}
L_{\mathrm{f}} & L_{\mathrm{i}} & \Lambda \\
M_{\mathrm{f}} & -M_{\mathrm{i}} & M
\end{array}\right) \\
& \times \int_{0}^{\infty} k^{2} \mathrm{~d} k C_{l}(k) \\
& \times\left[(-1)^{\lambda+M} H_{\lambda \lambda m}^{* \mathrm{fi}}\left(k, \Omega_{0}, v\right)+H_{l \lambda m}^{\mathrm{fi}}\left(k, \Omega_{0}, v\right)\right]
\end{aligned}
$$

where $\omega_{0}=\Omega_{0}+k v \cos \Theta_{k}^{\prime}+E_{\mathrm{i}}^{\mathrm{T}}$, and $\left(\hat{\omega}_{0}=\omega_{0}-E_{\mathrm{i}}^{\mathrm{T}}\right)$

$$
\begin{gathered}
H_{l \lambda m}^{\mathrm{fi}}\left(k, \Omega_{0}, v\right)=\int_{0}^{\infty} \mathrm{d} t \int_{-1}^{1} \mathrm{~d}\left(\cos \Theta_{k}^{\prime}\right) \mathrm{e}^{\mathrm{i} \hat{\omega}_{0} t} \\
\times G_{\lambda l}^{\mathrm{i}}\left(k, \omega_{0}, v t\right) Y_{L_{\mathrm{f}}}^{m}\left(\Theta^{\prime}, 0\right) Y_{l}^{m}\left(\Theta_{k}^{\prime}, 0\right) \\
\quad \times P_{\mathrm{f}}\left(\sqrt{k^{2}+v^{2}-2 k v \cos \Theta_{k}^{\prime}}\right)
\end{gathered}
$$

From this, the transfer probability from an initially occupied atomic shell is given by

$$
\begin{aligned}
P(\vartheta) & =2 \sum_{M_{\mathrm{f}} M_{\mathrm{i}}}\left|a_{\mathrm{fi}}\right|^{2}=8 \pi^{2} \sum_{\Lambda M}\left(Y_{\Lambda}^{M}(\vartheta / 2,0)\right)^{2} \\
& \times \mid \sum_{l \lambda M}(2 \lambda+1)^{1 / 2}\left\langle l\left\|Y_{\lambda}\right\| L_{\mathrm{i}}\right\rangle \\
& \times\left(\begin{array}{ccc}
l & \Lambda & L_{\mathrm{i}} \\
-m & 0 & m
\end{array}\right)\left(\begin{array}{ccc}
L_{\mathrm{f}} & L_{\mathrm{i}} & \Lambda \\
m & -m & 0
\end{array}\right) \\
& \times \int_{0}^{\infty} k^{2} \mathrm{~d} k C_{l}(k)\left[(-1)^{\lambda+M} H_{l \lambda m}^{* \mathrm{fi}}\left(k, \Omega_{0}, v\right)\right. \\
& \left.+H_{l \lambda m}^{\mathrm{fi}}\left(k, \Omega_{0}, v\right)\right]\left.\right|^{2}
\end{aligned}
$$

where it has been summed over the magnetic final substates.

This is the main result of this section. It shows that the angular distribution of the transfer probability between two angular momentum eigenstates is an incoherent sum of contributions from the partial waves $\Lambda$ where $\Lambda$ is restricted to $\left|L_{\mathrm{f}}-L_{\mathrm{i}}\right| \leqslant \Lambda \leqslant L_{\mathrm{f}}+L_{\mathrm{i}}$, and just determined by the spherical 
harmonics $Y_{\Lambda}^{M}(\vartheta / 2,0)$ which are multiplied by constant (i.e., $\vartheta$-independent) amplitudes containing the information on potentials and wavefunctions. This result, well-known from excitation and ionisation theories $[7,8]$ thus holds also if the initial and final states are centered at different nuclei. In contrast to the excitation case there is no parity selection rule, and no further restriction on $\Lambda$. The additional summations in eq. (4.4), compared to excitation theories (over $l, \lambda$ and $m$ ) describe the deformation of the final state wavefunction, as seen from the target system. It should be stressed that this (relatively) simple structure of the transfer probability is due to the form of eq. (2.10), as will be discussed in Section 6.

If the scattering angle $\vartheta$ becomes zero, which corresponds to the straight-line (zero impact parameter) case, only the $M=0$ contribution survives. As the $\vartheta$-independent amplitudes are identical for even $M$ (and fixed $\Lambda$ ) they are thus determined by the straight-line result. In order to make this more transparent, we introduce the Fourier transform $V(s)$ of the projectile field $V_{1}$ such that $v_{\lambda}(r, R)$ from eq. (3.4) can be written as

$v_{\lambda}(r, R)=4(2 \pi)^{1 / 2} \int_{0}^{\infty} s^{2} \mathrm{~d} s V(s) j_{\lambda}(s r) j_{\lambda}(s R)$

where $j_{\lambda}$ are spherical Bessel functions. The form factor in eq. (4.4) is given by

$$
\begin{gathered}
(-1)^{\lambda+M} H_{l \lambda m}^{* \mathrm{fi}}\left(k, \Omega_{0}, v\right)+H_{l \lambda m}^{\mathrm{fi}}\left(k, \Omega_{0}, v\right) \\
=\int_{-1}^{1} \mathrm{~d}\left(\cos \Theta_{k}^{\prime}\right) Y_{L_{\mathrm{f}}}^{m}\left(\Theta^{\prime}, 0\right) Y_{l}^{m}\left(\Theta_{k}^{\prime}, 0\right) \\
\quad \times P_{\mathrm{f}}\left(\sqrt{k^{2}+v^{2}-2 k v \cos \Theta_{k}^{\prime}}\right) B_{\lambda l M}
\end{gathered}
$$

with

$B_{\lambda l M}=\left\{(-1)^{\lambda+M} \int_{-\infty}^{0} \mathrm{~d} t+\int_{0}^{\infty} \mathrm{d} t\right\} G_{\lambda l}^{\mathrm{i}}\left(k, \omega_{0},|v t|\right) \mathrm{e}^{\mathrm{i} \hat{\omega}_{0} t}$

We recall that the factor $(-1)^{\lambda}$ for $t<0$ originates from $Y_{\lambda}^{\mu^{*}}(\mathbf{R})$ with $\mathbf{R}=\mathbf{v} t$. The time integral is easily carried out by using the relation

$$
\left(\frac{4 \pi}{2 \lambda+1}\right)^{1 / 2} j_{\lambda}(s R) Y_{\lambda}^{\mu^{*}}(\hat{\mathbf{R}})=\frac{1}{2} \mathrm{i}^{-\lambda} \int_{-1}^{1} \mathrm{~d}\left(\cos \Theta_{\mathrm{s}}\right)
$$$$
x \mathrm{e}^{\mathrm{i} s u t \cos \Theta_{\mathrm{s}}} P_{\lambda}\left(\cos \Theta_{\mathrm{s}}\right)
$$

which can be derived from the partial wave expansion of $\exp$ (isR). From the definition of $G_{\lambda l}^{\mathrm{i}}$, eq. (3.3), we then obtain:

$$
\begin{aligned}
B_{\lambda l M} & =\frac{4(2 \pi)^{1 / 2}}{v} \mathrm{i}^{\lambda} \int_{0}^{\infty} r^{2} \mathrm{~d} r R_{l}\left(k, \omega_{0}, r\right) R_{\mathrm{i}}(r) \\
& \times \int_{0}^{\infty} s \mathrm{~d} s V(s) j_{\lambda}(s r) \begin{cases}\pi P_{\lambda}\left(\hat{\omega}_{0} / s v\right) \Theta\left(s v-\left|\hat{\omega}_{0}\right|\right) & M \text { even } \\
2 \mathrm{i} Q_{\lambda}\left(\hat{\omega}_{0} / s v\right) & M \text { odd }\end{cases}
\end{aligned}
$$

where $\Theta$ is the step function and $P_{\lambda}$ and $Q_{\lambda}$ are the Legendre functions of the first and second kind, respectively. Thus there is a substantial difference between the form factors for even and odd $M$, which indicates that they may also be sensitive to different parts of the electronic wavefunctions. This result is again in complete analogy with the ionisation case.

\section{Calculation of the capture probability from $K$ and $L$ shell}

In this section we apply the results of the general theory to the transfer of a $K$ or $L$ electron into the 1s state of the light

Physica Scripta 26 projectile. We use the semiclassical impulse approximation which is obtained from eq. (2.10) by replacing the energy $\omega_{0}$ in the wavefunction $\left|\mathbf{k}, \omega_{0}\right\rangle^{\mathbf{T}}$ by its on-shell value $k^{2} / 2$, such that this wavefunction becomes a target continuum state $\left|\psi_{\mathbf{k}}^{\mathrm{T}}\right\rangle$. It should be noted that going on-shell is not entirely unproblematic due to the well-known discontinuities of the Coulomb functions. In general this may introduce inaccuracies that are far from negligible $[14,15]$, but if one can judge from the corresponding straight-line results, this approximation is not critical for the cases where large-angle data are currently available.

In order to evaluate the matrix elements it is more convenient not to use a partial wave expansion of the intermediate state $\left|\psi_{\mathbf{k}}^{\mathbf{T}}\right\rangle$ and the transition operator, as long as hydrogenic wavefunctions are used in the calculation. Instead of eqs. (3.4) and (4.5) we introduce the Fourier transform of the projectile field in the following way

$V_{1}(\mathbf{r}-\mathbf{R})=-\frac{Z_{1}}{2 \pi^{2}} \int \frac{\mathrm{d} \mathbf{s}}{\mathrm{s}^{2}} \mathrm{e}^{\mathrm{i} \mathbf{s}(\mathbf{r}-\mathbf{R})}$

where $Z_{1}$ is the projectile charge. Then, by making the coordinate transformation $\mathrm{q}_{0}=\mathrm{k}-\mathrm{s}$ the potential part of eq. (2.10) can be written as

$$
\begin{aligned}
a_{\mathrm{fi}}^{\mathrm{P}}= & \frac{\mathrm{i} Z_{1}}{2 \pi^{2}} \int \mathrm{d} \boldsymbol{q}_{0} \int \frac{\mathrm{d} \mathbf{s}}{s^{2}}\left\langle\psi_{\mathbf{q}_{0}+\mathbf{s}}^{\mathrm{T}}\left|\mathrm{e}^{\mathrm{isr}}\right| \psi_{\mathrm{i}}^{\mathrm{T}}\right\rangle \\
& \int_{-\infty}^{\infty} \mathrm{d} t \mathrm{e}^{\mathrm{i}\left(\Delta E-v^{2} / 2\right) t} \mathrm{e}^{\mathrm{i} \mathbf{q}_{0} \mathbf{R}} \varphi_{\mathrm{f}}^{\mathrm{P} *}\left(\mathrm{q}_{0}+\mathrm{s}-\dot{\mathbf{R}}\right)
\end{aligned}
$$

where $\Delta E=E_{\mathrm{f}}^{\mathrm{P}}-E_{\mathrm{i}}^{\mathrm{T}}$ and $\mathbf{R}$ is described by the broken-line path (3.5) with eq. (4.1). If $\left|\psi_{1}^{\mathrm{T}}\right\rangle$ is an $s$ state, it follows from eq. (4.2) with $L_{\mathrm{i}}=L_{\mathrm{f}}=0$ that the transition amplitude is independent of the scattering angle $\vartheta$ (as $\Lambda=0$ )

$a_{\mathrm{fi}}^{\mathrm{P}}(n s)=a_{\mathrm{fi}}^{\mathrm{SL}}(n s ; b=0), \quad n=1,2 \ldots$

and just given by the zero impact parameter straight-line result which is obtained from eq. (5.2) for $\vartheta=0$, i.e., $\mathbf{R}=v \mathrm{e}_{z}$.

For an initial $2 p, m=0$ state, we get from eq. (4.2) with $L_{\mathrm{i}}=\Lambda=1$ and $d_{00}^{(1)}(\vartheta / 2)=\cos \vartheta / 2$

$a_{\mathrm{fi}}^{\mathrm{P}}(2 p, m=0)=a_{\mathrm{fi}}^{\mathrm{SL}}(2 p, m=0 ; b=0) \cos \vartheta / 2$

where the amplitude is again determined by the straight-line result. The straight-line amplitudes are evaluated in [5] for the $K$-shell and [16] for the $L$-shell.

For an initial $2 p,|m|=1$ state, the transition amplitude can be written in the form (using that $d_{10}^{(1)}(\vartheta / 2)=2^{-1 / 2} \sin \vartheta / 2$ )

$a_{\mathrm{fi}}^{\mathrm{P}}(2 p,|m|=1)=a_{\mathrm{fi}}^{\mathrm{P}}(2 p,|m|=1 ; \vartheta=\pi) \sin \vartheta / 2$

As follows from the previous section, the amplitudes for odd $M$ can no longer be expressed by the straight-line results. Since the dependence on $\vartheta$ is known, it is sufficient to calculate (5.2) for $\vartheta=\pi$. Then $\mathbf{R}= \pm v t \mathrm{e}_{\mathrm{x}} \equiv \pm \mathbf{v} t$ (for $t \gtrless 0$ ), and the timeintegral is readily carried out, yielding ( $P$ denotes the principal value):

$$
\begin{aligned}
& a_{\mathrm{fi}}^{\mathrm{P}}(2 p,|m|=1 ; \vartheta=\pi)=-\frac{Z_{1}}{\pi^{2}} P \int \mathrm{dq}_{0} \int \frac{\mathrm{d} \mathbf{s}}{s^{2}} \\
& \quad \times\left\langle\psi_{\mathbf{q}_{0}+\mathbf{s}}^{\mathbf{T}}\left|\mathrm{e}^{\mathrm{iss}}\right| \psi_{\mathrm{i}}^{\mathrm{T}}\right\rangle \varphi_{\mathrm{f}}^{\mathrm{P}}\left(\mathrm{q}_{0}+\mathbf{s}-\mathbf{v}\right) \frac{1}{\Delta E-v^{2} / 2+\mathbf{q}_{0} \mathbf{v}}
\end{aligned}
$$


This result can also be derived independently from the previous section by using that the ionisation matrix element $[16,17]$

$$
\begin{gathered}
\left\langle\psi_{\mathbf{q}_{0}+\mathbf{s}}^{\mathrm{T}}\left|\mathrm{e}^{\mathrm{iss}}\right| \psi_{2 p, m= \pm 1}^{\mathrm{T}}\right\rangle= \pm \mathrm{i} \frac{Z_{2}^{7 / 2}}{2 \pi} M_{00}\left(M_{1} q_{0 \pm}-M_{2} s_{ \pm}\right) \\
M_{00}=\mathrm{e}^{\pi \eta / 2} \Gamma(1-\mathrm{i} \eta) \frac{\left[s^{2}-\left(k+\mathrm{i} Z_{2} / 2\right)^{2}\right]^{-\mathrm{i} \eta-1}}{\left[Z_{2}^{2} / 4+q_{0}^{2}\right]^{2-\mathrm{i} \eta}} \\
M_{1}=(1-\mathrm{i} \eta)\left[(2+\mathrm{i} \eta)+(2-\mathrm{i} \eta) \frac{s^{2}-\left(k+\mathrm{i} Z_{2} / 2\right)^{2}}{Z_{2}^{2} / 4+q_{0}^{2}}\right. \\
M_{2}=i \eta(1-i \eta)+(1+\mathrm{i} \eta)(2+\mathrm{i} \eta) \frac{Z_{2}^{2} / 4+q_{0}^{2}}{s^{2}-\left(k+\mathrm{i} Z_{2} / 2\right)^{2}}
\end{gathered}
$$

where $s_{ \pm}=2^{-1 / 2}\left(s_{x} \pm \mathrm{i} s_{y}\right), \eta=Z_{2} / k, Z_{2}$ the target charge and $k=\left|q_{0}+\mathbf{s}\right|$, changes sign when $\left(s_{ \pm}, q_{0 \pm}\right) \rightarrow-\left(s_{ \pm}, q_{0 \pm}\right)$ while the time-dependent part of the integrand in eq. (5.2) switches from the value for $t>0$ to the value for $t<0$ (and vice versa) under this operation, thus selecting the principal value part of the time integral.

For the further evaluation of eq. (5.6) we note that the terms in eq. (5.7) which are proportional to $s_{y}$ and $q_{0 y}$ vanish because the integrand is odd in these quantities.

We introduce spherical coordinates for $q_{0}$ and $s$, and choose $q_{0}$ as quantisation axis for $s$ and $e_{x}$ as quantisation axis for $q_{0}$. Then, $s_{x}=s\left(\cos \vartheta_{s, q_{0}} \cos \vartheta_{a_{0}}+\sin \vartheta_{s, q_{0}} \sin \vartheta_{q_{0}} \cos \varphi_{s}\right)$ and the integral over $\varphi_{s}$ can be performed analytically for a $1 s$ final state. The integral over $\varphi_{q_{n}}$ is trivial and we get

$$
\begin{aligned}
a_{\mathrm{fi}}^{\mathrm{P}}(2 p, m= \pm 1 ; \vartheta=\pi)=\mp \frac{4 \mathrm{i}}{\pi^{2}}\left(Z_{1} Z_{2}\right)^{7 / 2} \int_{0}^{\infty} q_{0}^{2} \mathrm{~d} q_{0} \\
\quad \times P \int_{-1}^{1} \mathrm{~d}\left(\cos \vartheta_{q_{0}}\right) \frac{1}{\Delta E-v^{2} / 2+q_{0} v \cos \vartheta_{q_{0}}} \\
\times \int_{0}^{\infty} \mathrm{d} s \int_{-1}^{1} \mathrm{~d}\left(\cos \vartheta_{s, q_{0}}\right) \frac{M_{00}}{\left(\alpha^{2}-\beta^{2}\right)^{3 / 2}} \\
\quad \times\left[M_{1} q_{0} \alpha \cos \vartheta_{q_{0}}-M_{2} s\left(\alpha \cos \vartheta_{s, q_{0}} \cos \vartheta_{q_{0}}\right.\right. \\
\left.\left.\quad-\beta \sin \vartheta_{s, q_{0}} \sin \vartheta_{q_{0}}\right)\right] \\
\alpha=Z_{1}^{2}+q_{0}^{2}+v^{2}+s^{2}-2 q_{0} v \cos \vartheta_{q_{0}}+2 q_{0} s \cos \vartheta_{s, q_{0}} \\
-2 v s \cos \vartheta_{q_{0}} \cos \vartheta_{s, q_{0}} \\
\beta=-2 v s \sin \vartheta_{q_{0}} \sin \vartheta_{s, q_{0}}
\end{aligned}
$$

This expression contains thus four integrals (i.e., one more than for the $m=0$ case) which have to be performed numerically. As there are several singularities in the integrand which makes the evaluation extremely time-consuming, we use an approximation that allows us to calculate the principal value integral analytically, exploiting that the main contribution to the transition amplitude comes from $\mathrm{q}_{0}$-values for which the energy denominator in eq. (5.8) vanishes. For a given $q_{0}$, we therefore replace $\cos \vartheta_{q_{0}}$ everywhere except in the energy denominator by the value

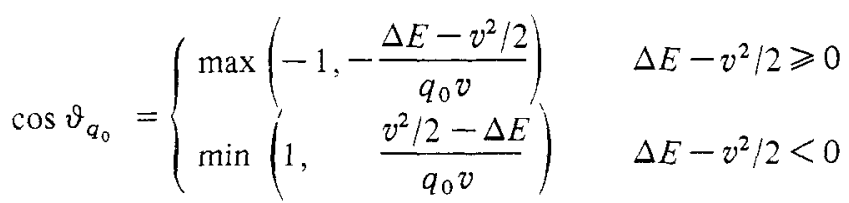

where the energy denominator has its smallest value. Numerical tests indicate that this approximation does not influence the numerical values of the transfer probabilities significantly. The principal value integral can then be carried out:

$$
\begin{aligned}
& P \int_{-1}^{1} \mathrm{~d}\left(\cos \vartheta_{q_{0}}\right) \frac{1}{\Delta E-\frac{1}{2} v^{2}+q_{0} v \cos \vartheta_{q_{0}}} \\
& \quad=\lim _{\epsilon \rightarrow 0} \frac{1}{2 q_{0} v} \ln \left[\frac{\left(\Delta E-v^{2} / 2+q_{0} v\right)^{2}+\epsilon^{2}}{\left(\Delta E-v^{2} / 2-q_{0} v\right)^{2}+\epsilon^{2}}\right]
\end{aligned}
$$

It remains to calculate the recoil contribution to the capture amplitude. Actually, the acceleration obtained from the brokenline path (3.5) with eq. (4.1), $\ddot{\mathbf{R}}=2 v \sin \vartheta / 2 \delta(t) \mathbf{e}_{x}$, is not proportional to $\mathbf{R}$, and thus not of the form (2.2) which holds for any central internuclear potential. In order to satisfy eq. $(2.2)$, we take instead $[7,8]$

$\ddot{\mathbf{R}}(t)=\lim _{\epsilon \rightarrow 0}\{\mathbf{v}(t)[\delta(t-\epsilon)-\delta(t+\epsilon)]\}$

such that an eq. (3.4)-type expansion holds for $V_{\mathrm{R}}(t)$ (with $\lambda=1$ ) and the results of the general theory can be applied.

In analogy to the case of the potential transition operator discussed above, we find that the recoil transition amplitude is proportional to the straight-line result for $s$ and $m=0$ states. Thus

$a_{\mathrm{fi}}^{\mathrm{R}}=0, \quad \mathrm{i}=1 s, 2 s, 2 p, m=0 \ldots$

as there is no recoil for a straight-line path. The only recoil contribution comes therefore from the $2 p,|m|=1$ initial state and has the form (4.2):

$a_{\mathrm{fi}}^{\mathrm{R}}(2 p,|m|=1)=a_{\mathrm{fi}}^{\mathrm{R}}(2 p,|m|=1 ; \vartheta=\pi) \sin \vartheta / 2$

Using the on-shell version of eq. (2.10) together with eqs. (2.2) and (5.11), the amplitude can be written as

$$
\begin{gathered}
a_{\mathrm{fi}}^{\mathrm{R}}(2 p,|m|=1 ; \vartheta=\pi)=\mathrm{i} v \frac{M_{1}}{M_{1}+M_{2}} \int \mathrm{d} \mathbf{k}\left\langle\psi_{\mathbf{k}}^{\mathrm{T}}|\times| \psi_{\mathrm{i}}^{\mathrm{T}}\right\rangle \\
\times\left[\varphi_{\mathrm{f}}^{\mathrm{P} *}(\mathbf{k}-\mathbf{v})+\varphi_{\mathrm{f}}^{\mathrm{P} *}(\mathbf{k}+\mathbf{v})\right]
\end{gathered}
$$

where $\mathbf{v}=v \mathbf{e}_{x}$ as before and the time integral has been performed by means of the $\delta$-functions. The dipole matrix element can be evaluated in terms of derivatives of the integral [17, p. 364]

$I=\int \mathrm{d} \mathbf{r} \mathrm{e}^{-\mathrm{i} \mathbf{k r}}{ }_{1} F_{1}(\mathrm{i} \eta, 1, \mathrm{i}(k r+\mathbf{k} \cdot \mathbf{r})) \mathrm{e}^{\mathrm{i} \mathbf{q} \cdot \mathbf{r}} \mathrm{e}^{-Z_{2} r / 2}$

with respect to $q$ and going to the limit $q \rightarrow 0$ afterwards. This leads for a $2 p, m=1$ initial state to

$$
\begin{gathered}
\left\langle\psi_{\mathrm{k}}^{T}|\mathrm{x}| \psi_{2 p, m= \pm 1}^{T}\right\rangle= \pm \frac{Z_{2}^{7 / 2}}{2^{3 / 2} \pi} \mathrm{e}^{\pi \eta / 2} \Gamma(1-\mathrm{i} \eta) \\
\times\left[k_{x}^{2} M_{x}(k)+M_{z}(k) \pm \mathrm{i} k_{x} k_{y} M_{x}(k)\right]
\end{gathered}
$$

$M_{x}(k)=2(1-i \eta)(2-i \eta) \frac{\left[-\left(k+i \frac{Z_{2}}{2}\right)^{2}\right]^{-i \eta-1}}{\left[Z_{2}^{2} / 4+k^{2}\right]^{3-i \eta}}$ 


$$
\begin{aligned}
& \times\left\{(2+\mathrm{i} \eta)-(3-\mathrm{i} \eta) \frac{\left(k+\mathrm{i} \frac{Z_{2}}{2}\right)^{2}}{Z_{2}^{2} / 4+k^{2}}\right\} \\
& M_{z}(k)=\frac{\left[-\left(k+\mathrm{i} \frac{Z_{2}}{2}\right)\right]^{-\mathrm{i} \eta-1}}{\left[Z_{2}^{2} / 4+k^{2}\right]^{2-i} \eta} \\
& \times\left\{(1+\mathrm{i} \eta)(2+\mathrm{i} \eta) \frac{Z_{2}^{2} / 4+k^{2}}{\left(k+\mathrm{i} \frac{Z_{2}}{2}\right)^{2}}-2\left(1+\eta^{2}\right)\right. \\
&\left.+(1-\mathrm{i} \eta)(2-\mathrm{i} \eta) \frac{\left(k+\mathrm{i} \frac{Z_{2}}{2}\right)^{2}}{Z_{2}^{2} / 4+k^{2}}\right\}
\end{aligned}
$$

where $\eta=Z_{2} / k$. Due to the symmetry property of the integrand, the term proportional to $k_{x} k_{y}$ vanishes. Introducing spherical coordinates for $\mathbf{k}$ and choosing $\mathbf{e}_{x}$ as quantisation axis, the angular integrals can be performed analytically for a $1 \mathrm{~s}$ final state. Thus one obtains

$$
\begin{aligned}
& a_{\mathrm{fi}}^{\mathrm{R}}(2 p, m= \pm 1 ; \vartheta=\pi)= \pm \frac{8 \mathrm{i}}{\pi} v Z_{1}^{5 / 2} Z_{2}^{7 / 2} \frac{M_{1}}{M_{1}+M_{2}} \\
& \quad \int_{0}^{\infty} k^{2} \mathrm{~d} k \mathrm{e}^{\pi \eta / 2} \Gamma(1-\mathrm{i} \eta)\left\{\frac{M_{z}(k)}{\left(Z_{1}^{2}+k^{2}+v^{2}\right)^{2}-4 k^{2} v^{2}}\right. \\
& +\frac{M_{x}(k)}{8 k v^{3}}\left[4 k v \frac{\left(Z_{1}^{2}+k^{2}+v^{2}\right)^{2}-2 k^{2} v^{2}}{\left(Z_{1}^{2}+k^{2}+v^{2}\right)^{2}-4 k^{2} v^{2}}\right. \\
& \left.\left.+\left(Z_{1}^{2}+k^{2}+v^{2}\right) \ln \frac{Z_{1}^{2}+(k-v)^{2}}{Z_{1}^{2}+(k+v)^{2}}\right]\right)
\end{aligned}
$$

The transition probability as function of the scattering angle then follows as the coherent sum of potential and recoil contribution

$$
P(\vartheta)=\left\{\begin{array}{l}
4 \sin ^{2} \vartheta / 2\left|a_{\mathrm{fi}}^{\mathrm{P}}(\vartheta=\pi)+a_{\mathrm{fi}}^{\mathrm{R}}(\vartheta=\pi)\right|^{2}, \quad \mathrm{i}=2 p,|m|=1 \\
2\left|a_{\mathrm{fi}}^{\mathrm{SL}}(b=0)\right|^{2}, \quad \mathrm{i}=1 s, 2 s \\
2 \cos ^{2} \vartheta / 2\left|a_{\mathrm{fi}}^{\mathrm{SL}}(b=0)\right|^{2}, \quad \mathrm{i}=2 p, m=0
\end{array}\right.
$$

where the sum over all initial electronic substates has been carried out.

\section{Numerical calculations and comparison with experiment}

We have evaluated the capture cross-section from the target $K$ shell and $L$ subshells into the projectile $K$ shell by means of the relations (5.18) with (5.8)-(5.10) and (5.17). Hydrogenic wavefunctions with Slater screening and experimental binding energies were used.

For the electron capture from He by protons, the theory predicts a scattering angle independent transition probability according to $(5.18)$ because the electrons are initially in a $1 s$ state and because the capture into higher projectile states (which would lead to a different angular distribution) can presumably be neglected for fast collisions. This is in good agreement with experiments on 0.2 and $0.4 \mathrm{MeV}(\mathrm{p}, \mathrm{He})$ collisions performed by Horsdal Pedersen and Loftager [6]. The absolute values of the transition probabilities were a factor four too low, which is not very surprising if the inadequacy of both the hydrogenic wavefunctions and a perturbative approach for $\mathrm{He}$ is taken into account. As will be argued below, the

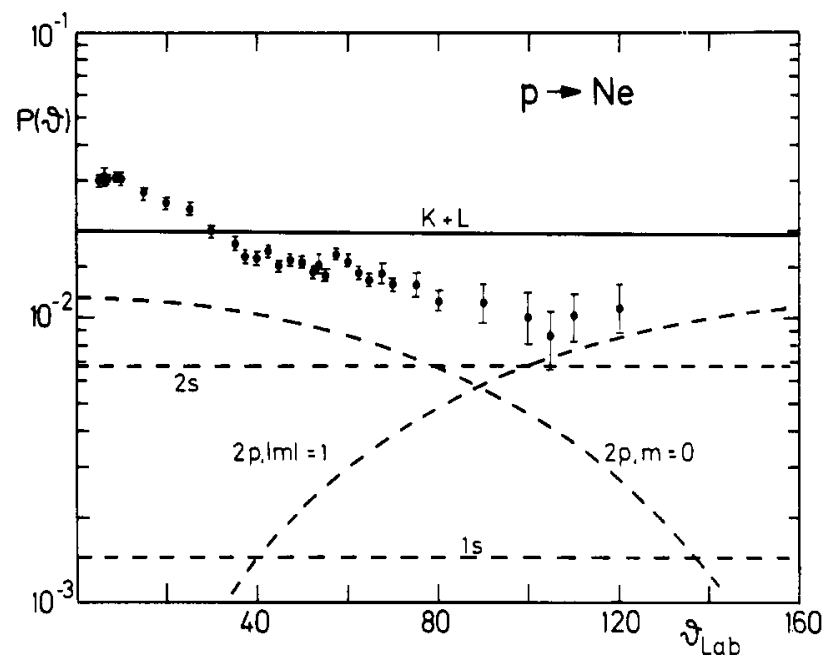

Fig. 2. Capture probability from the $K$ and $L$ shell of Ne in collisions with $0.4 \mathrm{MeV}$ protons as a function of scattering angle. The broken curves denote the capture probability from the $s$ and $p$ subshells, and the full curve is their sum. The experimental data are from [6].

prediction of a flat angular distribution for $K$-capture should, however, also be a feature of a non-perturbative approach.

Figure 2 shows the subshell capture probabilities from $\mathrm{Ne}$ in collisions with $0.4 \mathrm{MeV}$ protons. Apart from the constant contribution of the $s$-states, the transition probability at small scattering angles is mainly determined by the capture from the $2 p, m=0$ state while at large $\vartheta$ the $2 p,|m|=1$ initial state dominates. Thereby one should keep in mind that the quantisation axis was taken perpendicular to $\mathbf{R}$ at the distance of closest approach and not in the beam direction (which is the usual choice). The sum of all subshell contributions, however, is nearly independent of $\vartheta$, and we found this behaviour for all projectile energies considered $(0.2-0.75 \mathrm{MeV})$. A comparison with the experimental data shows that although the absolute value of the transition probability can be reasonably well reproduced, the angular dependence is much stronger than predicted by theory, and follows rather the $\cos ^{2} \vartheta / 2$-dependence of the $2 p, m=0$ capture probability.

In Fig. 3 similar calculations for $1 \mathrm{MeV}$ proton impact on Ar are presented. The experimental results are total capture probabilities, but experimental investigations both of total Ar capture cross-sections [18], as well as of capture probabilities for $0-10^{\circ}$ scattering $[3,6]$ indicate that capture from the $L$-shell dominates the transfer probability. Our calculations show that transitions from the $2 p, m=0$ states are much more important than capture from the $|m|=1$ states, leading to a much steeper dependence of the total transfer probability with scattering angle than for $\mathrm{Ne}$. The recoil term increases the $2 p,|m|=1$ probability with $15 \%$ and $30 \%$ for $\mathrm{Ne}$ and $\mathrm{Ar}$, respectively.

As is evident from Figs. 2 and 3, although the present theory reproduces the magnitude of the $K+L$ capture probabilities reasonably well, the experimental angular dependence is much stronger than the theoretical, in particular at the smaller scattering angles. Several reasons for these discrepancies can be suggested.

(i) Wavefunction effects. The $L$-shells of Ar and, in particular, $\mathrm{Ne}$ are not well represented by hydrogenic wavefunctions, and the use of more realistic wavefunctions can influence the tran. sition matrix element considerably, as in the case of ionisation 


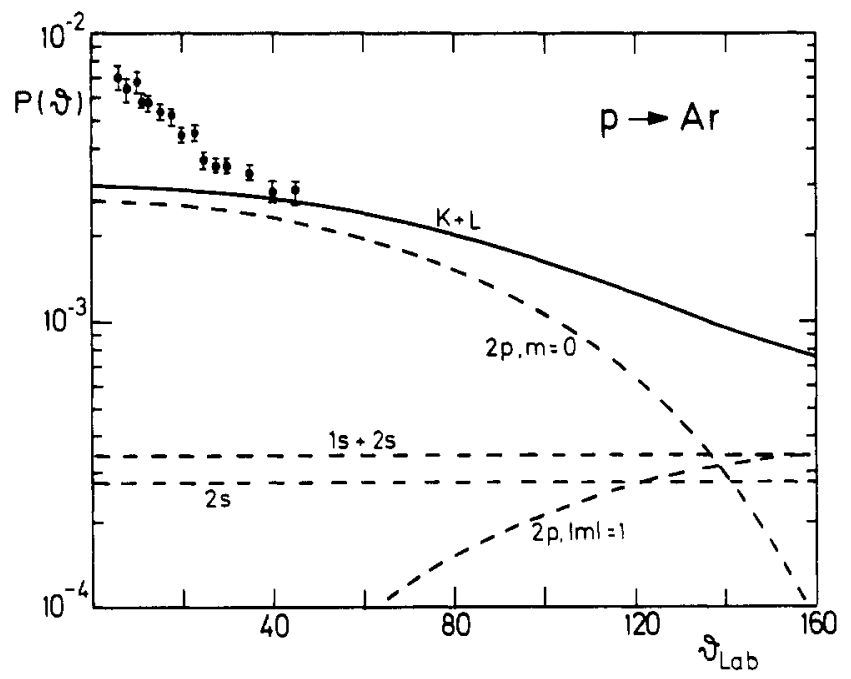

Fig. 3. Capture probability from the $K$ and $L$ shell of Ar in collisions with $1 \mathrm{MeV}$ protons as a function of scattering angle. The broken curves denote the capture probability from the $s$ and $p$ subshells, and the full curve is their sum. The experimental data are from [6].

[19]. Since the transition operators for even and odd $m$ weigh different parts of the wavefunctions differently, this can also influence the angular distributions. However, as long as only capture to the ground state is considered, the transfer prob. ability will still have the structure

$P(\vartheta)=a_{1}+a_{2} \cos ^{2} \frac{\vartheta}{2}+a_{3} \sin ^{2} \frac{\vartheta}{2}$

with $a_{1}, a_{2}, a_{3}$ all $\geqslant 0$. Thus $P(\vartheta)$ cannot vary on an angular scale smaller than $90^{\circ}$ in this case, in contrast to the apparent experimental behaviour.

(ii) Capture to excited states. From eq. (4.4) it follows that capture into states with $L_{\mathbf{f}}>0$ will introduce additional multi. pole contributions in the angular distributions. For target $K$. capture most theories seem to agree with the Brinkman-Kramers result that capture into the projectile ground state dominates [20] (cf. also [17]). For capture from higher-lying states, in particular with $L_{\mathrm{i}}>0$, much less is certain. Unfortunately the present approach is not easily extended to these cases, but a calculation of the $2 p, m=0 \rightarrow 2 p, m=0$ probability for $\mathrm{Ne}$ gave a negligible contribution. This seems to indicate that transfer to excited states is not very important, but it should be borne in mind that contributions from very many final states might still add up to a non-negligible contribution.

(iii) Restrictions on the theory. The present approach is based on perturbation theory and is thus not expected to be valid for systems with initial and final states belonging to similar effective charges, like $\mathrm{He} K$-shell or Ne $L$-shell capture into the hydrogen ground state, except possibly at very high energies. In this case one may think of using a symmetric version of the impulse approximation, as suggested by Briggs [21] which treats the projectile and the target field on equal footing. Actually our general result for the structure of the angular distributions, as expressed in eq. (4.4), is somewhat more general than the impulse approximation. It follows from introducing the broken-line approximation in eq. (2.10), irrespectively of what kind of state $\left|\mathbf{k}, \omega_{0}\right\rangle^{\mathbf{T}}$ is taken to be. In particular, if this state is replaced by a plane wave (and $\omega_{0}=$ $\frac{1}{2} k^{2}$ ), the same structure for the angular distribution is obtained in the broken-line version of the Brinkman-Kramers theory.
The general form of eq. (2.10) is valid if the transfer process can be adequately described by a target excitation immediately followed by an overlap with a Galilei-transformed projectile state. However, if one starts from the expression (2.7) instead, the angular distributions will be modified, because the scattering angle dependence entering through $\dot{\mathbf{R}}\left(t^{\prime}\right)$ in $\varphi_{\mathrm{f}}^{\mathrm{P}}$ will no longer add coherently to the one coming from $V_{1}(t)$ when $t \neq t^{\prime}$. In the present case this modification can hardly account for the discrepancies between theory and experiment, but a similar situation will also arise if one iterates eq. (2.6) to higher orders in the weak potential. Then the angular distributions from the potential terms of different times will not in general add coherently, and the situation can be complicated further by the timedependence of the overlap term. Thus, the projectile scattering angle dependence for collision systems where higher-order contributions are non-negligible may deviate significantly from the one predicted from eq. (4.4). Such corrections should be most important for transitions between states of angular momentum different from zero. On the other hand, if $L_{\mathrm{i}}=$ $L_{f}=0$, the intermediate states will also to a large extent be $l=0$ states, and any averaging process will also tend to wash out structures arising if $l \neq 0$. Thus for such situations the transfer probability will tend to remain independent of scattering angle even if higher-order processes are important.

\section{Conclusion}

We have extended the semiclassical theory for charge transfer in asymmetric ion-atom collisions to allow for a curved trajectory for the internuclear motion. Using a first-order expansion of the scattering amplitude in terms of the weak potential and approximating the classical path by a zero impact parameter broken-line path, the dependence of the transfer probability on the projectile scattering angle has been expressed as partial wave sum, eq. (4.4). In spite of the fact that the initial and final states are centered on different nuclei, only partial waves consistent with the initial and final states being centered on the same nucleus contributes to this sum. This simple form allows for definite predictions of the overall structure of the projectile scattering angle dependence for a given transfer process.

The formalism has been applied to the calculation of capture into the hydrogen ground state from $\mathrm{He}$ and $\mathrm{Ne}$ and from the Ar $K$ and $L$ shells using the impulse approximation. For He the angular distribution is in good agreement with recent experiments, although the absolute magnitude is not. For $\mathrm{Ne}$ and $\mathrm{Ar}$ the calculated probabilities are in reasonable agreement with experiments, but the angular distributions are too flat, in particular at the smaller scattering angles. The origin of these discrepancies is unclear.

The present theory can probably be improved by using more accurate wavefunctions. Furthermore, the impulse approximation is not really applicable in some of the cases we have considered, like for $\mathrm{He}$ and the $\mathrm{Ne} L$-shell, and improved calculations are necessary. New experimental results would be very welcome, in particular if more information on the initial and final states of the transferred electron could be obtained.

\section{Acknowledgment}

We should like to thank E. Horsdal Pedersen for directing our interest to this field and for providing us with unpublished data. 


\section{References}

1. Cocke, C. L., Macdonald, J. R., Curnutte, B., Varghese, S. L. and Randall, P., Phys. Rev. Lett. 36, 782 (1976).

2. Bratton, T., Cocke, C. L. and Macdonald, J.R., J. Phys. B10, L517 (1977).

3. Horsdal Pedersen, E., Invited talk at the XII ICPEAC, Gatlinburg (1981) (to be published).

4. Briggs, J. S., J. Phys. B10, 3075 (1977).

5. Jakubaßa-Amundsen, D. H. and Amundsen, P. A., Z. Phys. A297, 203 (1980).

6. Horsdal Pedersen, E. and Loftager, P., XII ICPEAC, Gatlinburg (1981); Book of Abstracts of Contributed Papers (Edited by S. Datz), Vol. 2, p. 656 (1981) (and to be published).

7. Ciocchetti, G. and Molinari, A., Nuovo Cim. B40, 69 (1965).

8. Andersen, J. U., Kocbach, L., Laegsgaard, E., Lund, M. and Moak, C. D., J. Phys. B9, 3247 (1976).

9. Edmonds, A. R. Drehimpulse in der Quantenmechanik. Mannheim, BI (1964).
10. Amundsen, P. A., J. Phys. B11, 3197 (1978).

11. Dettmann, K., Springer Tracts in Modern Physics 58, 119 (1971).

12. Bang, J. and Hansteen, J.M., Kgl. Danske Vidensk. Selsk., Mat. -Fys. Meddr 31, No. 13, 1 (1959)

13. Chemin, J. F., Anholt, R., Stoller, Ch., Meyerhof, W. E. and A mundsen, P. A., Phys. Rev. A24, 1218 (1981).

14. Macek, J. and Taulbjerg, K., Phys. Rev. Lett. 46, 170 (1981).

15. Jakubaßa-Amundsen, D. H. and Amundsen, P. A., J. Phys. B14, L705 (1981).

16. Jakubaßa-Amundsen, D. H., J. Phys. B14, 2647 (1981),

17. McDowell, M. R. C. and Coleman, J. P., Introduction to the Theory of Ion-Atom Collisions, Chapters 6-8. North Holland, Amsterdam (1970).

18. Rфdbro, M., Horsdal Pedersen, E., Cocke, C. L. and Macdonald, J. R., Phys. Rev. A19, 1936 (1979).

19. Aashamar, K. and Amundsen, P. A., J. Phys. B14, 483 (1981).

20 Nikolaev, V. S., Sov. Phys. JETP 24, 847 (1967)

21. Briggs, J. S., J. Phys. B13, L717 (1980). 